This item was submitted to Loughborough's Research Repository by the author.

Items in Figshare are protected by copyright, with all rights reserved, unless otherwise indicated.

\title{
Closed-loop extended orthogonal space time block coding for four relay nodes under imperfect synchronization
}

PLEASE CITE THE PUBLISHED VERSION

PUBLISHER

(c) IEEE

VERSION

VoR (Version of Record)

LICENCE

CC BY-NC-ND 4.0

\section{REPOSITORY RECORD}

Elazreg, A.M., and Jonathon Chambers. 2019. "Closed-loop Extended Orthogonal Space Time Block Coding for Four Relay Nodes Under Imperfect Synchronization”. figshare. https://hdl.handle.net/2134/5551. 
This item was submitted to Loughborough's Institutional Repository (https://dspace.lboro.ac.uk/) by the author and is made available under the following Creative Commons Licence conditions.

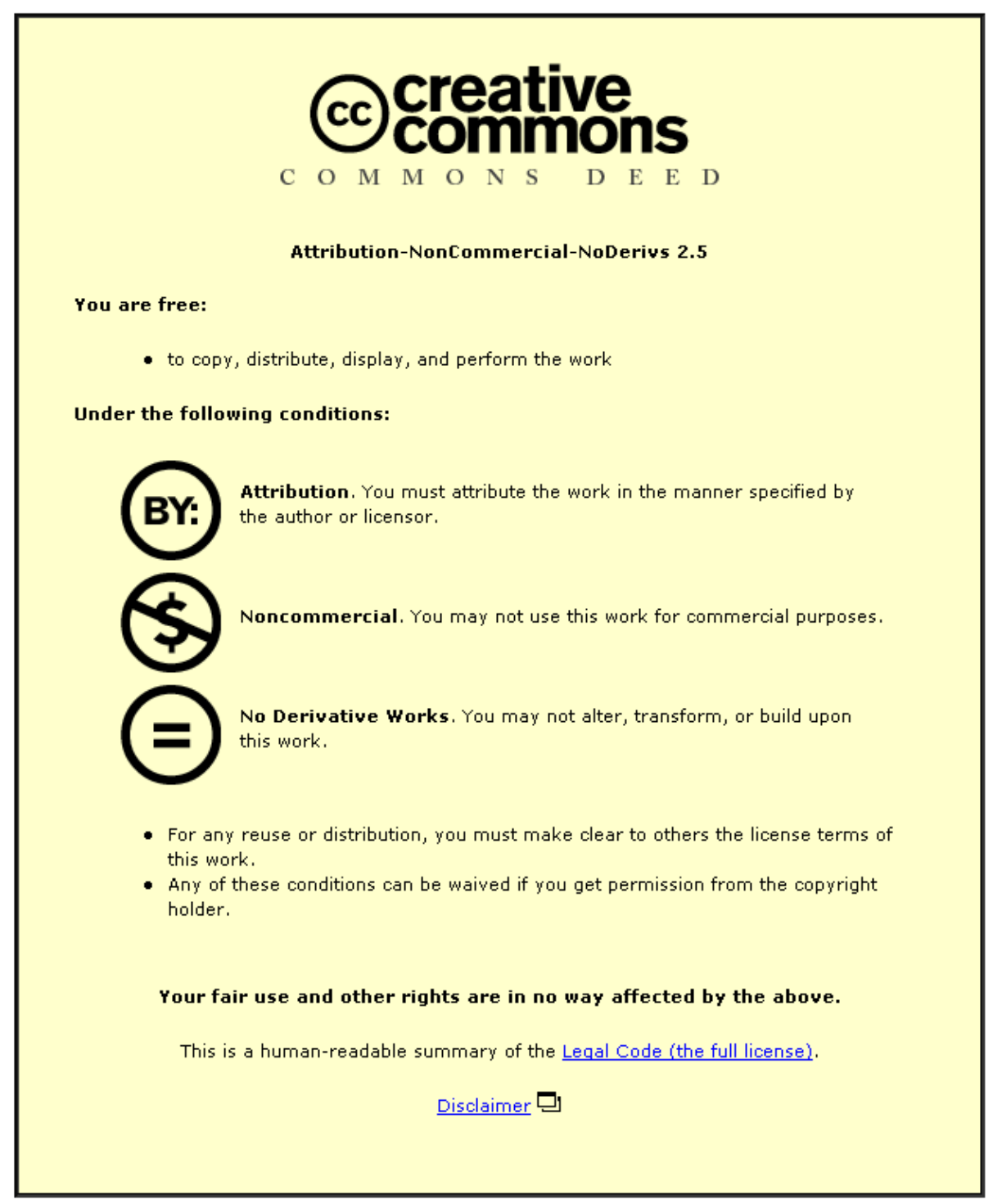

For the full text of this licence, please go to: http://creativecommons.org/licenses/by-nc-nd/2.5/ 


\title{
CLOSED-LOOP EXTENDED ORTHOGONAL SPACE TIME BLOCK CODING FOR FOUR RELAY NODES UNDER IMPERFECT SYNCHRONIZATION
}

\author{
A.M.Elazreg, and J.A.Chambers \\ Advanced Signal Processing Group \\ Electronic and Electrical Engineering Department \\ Loughborough University, Leicestershire, UK,LE11 3TU \\ Email:\{A.Elazreg, J.A.Chambers\}@1boro.ac.uk
}

\begin{abstract}
In future collaborative wireless communication systems with high data rate, interference cancellation is likely to be required in cooperative networks at the symbol level to mitigate synchronization errors. In this paper, we therefore examine closed-loop extended orthogonal space time block coding (CL EO-STBC) for four relay nodes and apply parallel interference cancellation (PIC) detection scheme to mitigate the impact of imperfect synchronization. Simulation results illustrate that the closed-loop EO-STBC scheme under imperfect synchronization can achieve good performance with simple linear processing and outperform previous methods. Moreover, a PIC scheme is shown to be very effective in mitigating impact of imperfect synchronization with low structural and computational complexity.
\end{abstract}

Index Terms - Closed-loop extended orthogonal space time block coding, linear processing, partial feedback and parallel interference cancellation detection.

\section{INTRODUCTION}

One of the most common techniques in wireless communication systems with multiple antennas at transmitter is the orthogonal space time block coding STBC which is popularly known as transmit diversity (TD). Furthermore, it is the most significant contributor for achieving transmitter diversity with full data rate, due to its high diversity order and low decoding complexity [1][2]. Due to the cost, size limitation and hardware complexity, it is usually difficult to have multiple antennas in one mobile communication node. In recent years, distributed space time block coding (D-STBC) has became an active and important area of research in wireless communication by sharing antennas of different users since virtual multiple antenna transmitters are generated to improve reliability of transmission and achieve transmitter diversity by applying (STBC) [3][4][5].

In (D-STBC) most widely researchers have focused on the case of two relay nodes using the Alamouti scheme [1] and assumed that cooperative relay nodes are perfectly synchronized at the symbol level, that means all transmitted symbols from all relay nodes reach their destination node at the same time. Unfortunately, this is not a true representation of the real transmission environment, practically the assumption of accurate symbol level synchronization in wireless communication systems ( such as ad hoc networks), is difficult or even impossible to achieve due to the signalling overhead [6][7]. In the imperfect synchronization case between the relay nodes, the channel might become dispersive even under flat fading condition which will damage the orthogonality of (STBC) and can lead to essential performance impairment [8]. Previously, there has been limitated work reported regarding the mitigation of interference at the symbol level. The equalization technique was mainly used at the destination node to perform such mitigation [6][9]. Some other techniques were also used to overcome the problem of signal synchronization. One of which proposing a new STBC detection solution based on PIC detection [8][10]. In the case of four relay nodes under imperfect synchronization which was considered in [10], the maximum symbol transmission rate by using complex orthogonal space time block coding was 3/4 and the PIC detector has moderate computational complexity. However, in this paper we propose a closed-loop EO-STBC [11] under imperfect synchronization with low structural and computational complexity in the PIC detector. We show that closed-loop EO-STBC can achieve full data rate with simple detection, contrary to what was previously achieved in [10].

The paper is organized as follows, Section II describes the proposed closed-loop extended-orthogonal space time block coding under imperfect synchronization. To mitigate the interference at the symbol level at the destination node, the full scheme of parallel interference cancelation (PIC) is introduced in Section III. Simulation results are presented in Section IV. The conclusion follow in Section V. In the remaining part of this paper, [.] $]^{T},[.]^{*},||,. \Re\{$.$\} and \{.\}^{H}$ denote "transpose", "conjugate","absolute value", "real part of complex number" and "Hermitian (complex conjugate transpose) of a matrix", respectively. $C N\left(0, \sigma^{2}\right)$ represent a Gaussian distributed complex number with the standard variance of $\sigma^{2}$ (i.e. $0.5 \sigma^{2}$ per dimension).

\section{CLOSED-LOOP EO-STBC UNDER IMPERFECT SYNCHRONIZATION}

In this paper the four relays mode closed-loop EO-STBC is depicted in both figures Fig. 1 and Fig. 2, comprising the source node (S), the destination node (D), the four relay nodes $\left(R_{1}, R_{2}, R_{3}, R_{4}\right)$ and the two feedback bits. The scheme comprises two phases. Phase one Fig. 1 for broadcasting and phase two Fig. 2 for relaying. 
Phase 1: Node $\mathrm{S}$ transmits while nodes (D) and $R_{m}$ receive. The EO-STBC is encoded at $R_{m}$ where $m=1,2, . ., 4$ for phase 2, at the $\mathrm{S}$ node data symbols are grouped into two symbols $s(i)=$ $[s(1, i), s(2, i)]^{T}$ and then transmitted to the $\mathrm{D}$ and $R_{m}$ nodes as shown in Fig. 1. The received signal at $D$ node after passing through

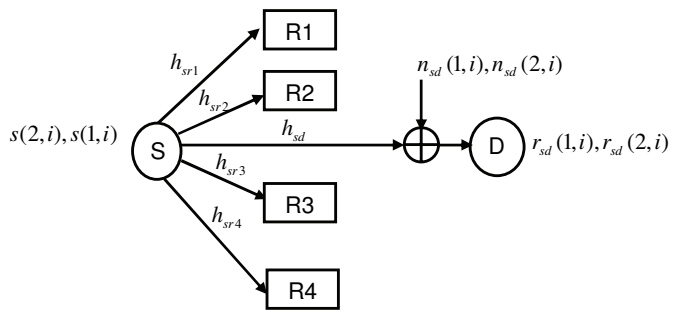

Fig. 1. Phase 1 relay mode with four relay nodes and a direct path

the channels $h_{s d}$ can be expressed as

$$
r_{s d}(i)=s(i) h_{s d}+n_{s d}(i)
$$

where $h_{s d} \in C N\left(0, \sigma^{2}\right)$ is the channel gain between $\mathrm{S}$ and $\mathrm{D}$, $r_{s d}(i)=\left[r_{s d}(1, i), r_{s d}(2, i)\right]^{T}, n_{s d}(i)=\left[n_{s d}(1, i), n_{s d}(2, i)\right]^{T}$, where $n_{s d}(j, i) \in C N\left(0, \sigma_{n}^{2}\right)$ is additive Gaussian noise. The least squares (LS) method can be used to detect which symbols were actually transmitted.

$$
\hat{s}_{s d}(j, i)=\arg \left\{\left.\left.\min _{S_{l} \in S}\left|h_{s d}^{*} r_{s d}(j, i)-\right| h_{s d}\right|^{2} S_{l}\right|^{2}\right\}
$$

Phase 2: Shown in Fig. 2, at the relay nodes $R_{m}$, the data packet is encoded, and then the modulated symbols are fed into the EO-STBC [11]. The relay mode of decode and forward is used in this paper. As such, a sufficient level of cyclic redundancy check (CRC) can be included into the data packet at $\mathrm{S}$ node, therefore the relaying will happen if the date packet is correctly detected at $R_{m}$. This arrangement is termed "selective relaying" in [12].

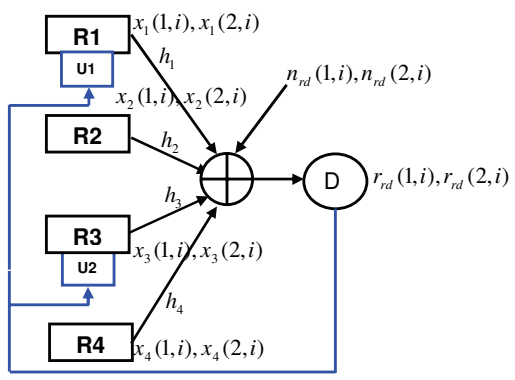

Fig. 2. Phase 2 relay mode with four relay nodes together with feedback scheme to achieve full diversity.

Denoting the encoding data packet at $R_{m}$ corresponding to $s(i)$ as $x_{m}(i)=\left[x_{m}(1, i), x_{m}(2, i)\right]^{T}$. The EO-STBC matrix which is transmitted by $R_{m}$ is therefore

$$
\begin{aligned}
& {\left[\begin{array}{rrrr}
x_{1}(j, i) & x_{2}(j, i) & x_{3}(j, i) & x_{4}(j, i)
\end{array}\right]=} \\
& {\left[\begin{array}{rrrr}
s(1, i) & s(1, i) & s(2, i) & s(2, i) \\
-s^{*}(2, i) & -s^{*}(2, i) & s^{*}(1, i) & s^{*}(1, i)
\end{array}\right]}
\end{aligned}
$$

For achieving full diversity, the data packet from $R_{1}$ is multiplied by
$U_{1}=(-1)^{a}$ and $R_{3}$ is multiplied by $U_{2}=(-1)^{b}$ as shown in Fig. 2 , where $a, b=0,1$ prior to transmitting them to $\mathrm{D}$ node. In this paper $a$ and $b$ are two feedback parameters which are determined by channel condition, When $a$ or $b=1$ then $U_{1}$ or $U_{2}$ equal -1 . That means the data packet from $R_{1}$ and $R_{3}$ in (3) will be phase rotated by $180^{\circ}$ before transmitting. Otherwise, the data packet can be directly transmitted. Due to the imperfect synchronization such as different propagation delay, $x_{m}(i)$ signals will not arrive at the D node in the same time. Which means accurate synchronization is difficult or impossible [7]. As shown in Fig 3 there is normally a timing misalignment of $\tau_{m}$ among the received versions of these signals. Since a rough synchronization is always required. At this point $\tau_{m}$ is smaller than sample period $T$ [7], as shown in Fig 3. It will still cause intersymbol interference (ISI) from neighboring symbols at D node, owing to sampling or matched filtering (whatever pulse shaping is used). As shown in Fig. 3, we assume that the received signal at

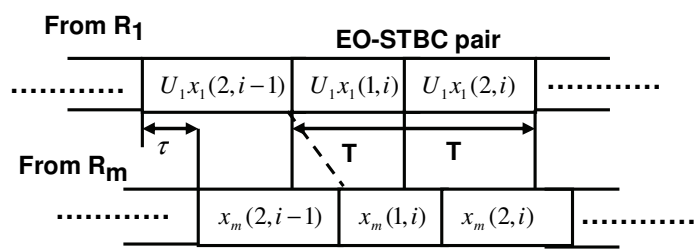

Fig. 3. Imperfect synchronization at the D node the received signal at $\mathrm{D}$ node is the superposition of four symbol.

the D node is perfectly synchronized to $R_{1},\left(\tau_{1}=0\right)$ therefore, the received signals $r_{r d}(1, i)$ and $r_{r d}(2, i)$ at the D node in the two independent time intervals are expressed as follows

$$
\begin{aligned}
r_{r d}(1, i)= & U_{1} h_{1}(0) x_{1}(1, i)+h_{2}(0) x_{2}(1, i)+U_{2} h_{3}(0) \\
& x_{3}(1, i)+h_{4}(0) x_{4}(1, i)+h_{2}(-1) x_{2}(2, i-1) \\
+ & U_{2} h_{3}(-1) x_{3}(2, i-1)+h_{4}(-1) x_{4}(2, i-1) \\
+ & n_{r d}(1, i) \\
& \\
r_{r d}(2, i)= & U_{1} h_{1}(0) x_{1}(2, i)+h_{2}(0) x_{2}(2, i)+U_{2} h_{3}(0) \\
& x_{3}(2, i)+h_{4}(0) x_{4}(2, i)+h_{2}(-1) x_{2}(1, i) \\
+ & U_{2} h_{3}(-1) x_{3}(1, i)+h_{4}(-1) x_{4}(1, i) \\
+ & n_{r d}(2, i)
\end{aligned}
$$

where $n_{r d}(j, i) \in C N\left(0, \sigma_{n}^{2}\right)$ is additive Gaussian noise and $h_{m}(l)$ is the channel gains between $R_{m}$ and D node under imperfect inter node synchronization and assumed to be block Rayleigh fading from packet to packet,(note that $\left.h_{1}(0)=h_{1}\right)$ i.e $h_{m} \in C N\left(0, \sigma_{r}^{2}\right)$. For fair comparison with non-relay scheme, all relay nodes transmit at $1 / 4$ power i.e $\sigma_{r}^{2}=\sigma_{s}^{2} / 4$. Due to imperfect synchronization $h_{m}(-1)$ reflects the inter symbol interference from previous symbol. The relative strength of $h_{m}(-1)$ will be expressed by ratio as follows [8][10].

$$
\beta_{m}=\left|h_{m}(-1)\right|^{2} /\left|h_{m}(0)\right|^{2}
$$

where $\beta_{m}$ is used to denote how the level of imperfect synchronization. Similarly, the equation $\left|h_{m}(-1)\right|^{2}+\left|h_{m}(0)\right|^{2} \leq\left|h_{m}\right|^{2}$ was used and we normally have $\beta_{m}=0$ for $\tau_{m}=0$, and $\beta_{m}=1$ (i.e. $0 \mathrm{~dB}$ ) for $\tau_{m}=0.5 T$. Substituting (3) into (4) and (5), the received 
signals $r_{r d}(1, i)$ and $r_{r d}(2, i)$, conjugated for convenience at two independent time intervals are expressed as follows

$$
\mathbf{r}(i)=\mathbf{H} s(i)+\mathbf{I}(i)+\mathbf{n}_{r d}(i)
$$

where

$$
\begin{gathered}
\mathbf{r}(i)=\left[r_{r d}(1, i), r_{r d}^{*}(2, i)\right]^{T}, \mathbf{n}_{r d}(i)=\left[n_{r d}(1, i), n_{r d}^{*}(2, i)\right]^{T}, \\
\mathbf{H}=\left[\begin{array}{cc}
U_{1} h_{1}(0)+h_{2}(0) & U_{2} h_{3}(0)+h_{4}(0) \\
U_{2}^{*} h_{3}^{*}(0)+h_{4}^{*}(0) & -U_{1}^{*} h_{1}^{*}(0)-h_{2}^{*}(0)
\end{array}\right],
\end{gathered}
$$

and

$$
\begin{gathered}
\mathbf{I}(1, i)=h_{2}(-1) s^{*}(2, i-1)+\left(U_{2} h_{3}(-1) h_{4}(-1)\right) s^{*}(1, i-1) \\
\mathbf{I}^{*}(2, i)=h_{2}^{*}(-1) s^{*}(1, i)+\left(U_{2}^{*} h_{3}^{*}(-1) h_{4}^{*}(-1)\right) s^{*}(2, i)
\end{gathered}
$$

from (7), the conventional closed-loop EO-STBC detection can be carried out via the following standard two step procedure assuming perfect channel state information at $\mathrm{D}$ node. Therefore, the detection vector $\mathbf{y}(i)=[y(1, i), y(2, i)]^{T}$ with the receive vector $\mathbf{r}(i)=\left[r_{r d}(1, i), r_{r d}^{*}(2, i)\right]^{T}$ can be calculated

STEP 1: linear transform

$$
\begin{aligned}
\mathbf{y}(i) & =[y(1, i), y(2, i)]^{T}=\mathbf{H}^{H} \mathbf{r}(i) \\
& =\underbrace{\left(\left|U_{1}\right|^{2}\left|h_{1}(0)\right|^{2}+\left|h_{2}(0)\right|^{2}+\left|U_{2}\right|^{2}\left|h_{3}(0)\right|^{2}+\left|h_{4}(0)\right|^{2}\right.}_{g_{c}} \\
& +\underbrace{\left.2 \Re\left(U_{1} h_{1}(0) h_{2}^{*}(0)\right)+2 \Re\left(U_{2} h_{3}(0) h_{4}^{*}(0)\right)\right)}_{g_{f}} s(i) \\
& +\mathbf{H}^{H} \mathbf{I}(i)+\mathbf{v}(i)
\end{aligned}
$$

where $\mathbf{v}(i)=\mathbf{H}^{H} \mathbf{n}_{r d}(i)$ is a noise component. Since $\left|U_{1}\right|^{2}=$ $\left|U_{2}\right|^{2}=1$ then the channel gain $g$ is expressed as follows

$$
g=\sum_{i=1}^{4} \underbrace{\left|h_{i}(0)\right|^{2}}_{g_{c}}+\underbrace{2 \Re\left(h_{1}(0) h_{2}^{*}(0)+h_{3}(0) h_{4}^{*}(0)\right)}_{g_{f}}
$$

where $g_{c}$ is the conventional channel gain for four relay nodes and $g_{f}$ is the feedback performance gain. The $g_{f}$ changes with the defined value of $U_{1}$ and $U_{2}$ as mention above. According to the above analysis, the two-bit feedback can be designed as shown in [11], in which each element of feedback performance gain $g_{f}$ in (9) must be nonnegative. The two-bit feedback has been chosen to maximize the value of $g_{f}$ in (8), which leads to a larger received SNR, which shows the closed-loop EO-STBC scheme achieves the better performance rather than the pervious work in [10] under imperfect synchronization. On the other hand, closed-loop EO-STBC can be used for more than four relay node under imperfect synchronization. However, more feedback bit needs for large relay nodes.

STEP 2: :Least squares (LS) detection

$$
\hat{s}_{r d}(j, i)=\arg \left\{\left.\left.\min _{S_{l} \in S}|y(j, i)-| g\right|^{2} S_{l}\right|^{2}\right\}
$$

The above procedure can suffer from significant detection error, because of the $\mathbf{H}^{H} \mathbf{I}(i)$ component in (8) which will damage the orthogonality of closed-loop EO-STBC. However, this procedure can give good performance gain when the $h_{m}(-1)=0$ (i.e. $\tau=0$ in case of perfect synchronization), thats why the performance of conventional closed-loop EO-STBC detector impairment under imperfect synchronization.

\section{PIC BASED DETECTION}

The principle of parallel interference cancelation is applied to remove the impact of imperfect synchronization of the interference component $\mathbf{I}(i)$ from (7), assuming perfect channel state information at D node. Whether the interference component $\mathbf{I}(i)$ in (7) is removed, that means the PIC detection would achieve maximum likelihood due to the closed-loop EO-STBC structure. Previous, PIC detection is applied to a co-located STBC system [13]. Since that $x_{m}(2, i-1)$ in (4) and (5) is already known if the detection process has been initialized properly, $I(1, i)$ can be removed during the initialization stage. The PIC iteration process can then be used to mitigate the impact of $\mathbf{I}(i)$ in (7) as follows

Initialization : 1 -set iteration number $\mathrm{K}=0$

2- From the received signal $\mathbf{r}(i)$ in (7) calculate

$$
\dot{\mathbf{r}}^{(0)}(i)=\left[\mathbf{r}_{r d}(1, i)-\mathbf{I}(1, i), \mathbf{r}_{r d}^{*}(2, i)\right]^{T}
$$

3- Because of the poor performance of the conventional detector, the (DT) detection result in (2) has been used to initialize $s^{(0)}(i)$ :

$$
s^{(0)}(i)=\left[s^{(0)}(1, i), s^{(0)}(2, i)\right]^{T}=\left[\hat{s}_{s d}^{(0)}(1, i), \hat{s}_{s d}^{(0)}(2, i)\right]^{T}
$$

4- Set the iteration number $\mathrm{K}=1,2, \ldots . \mathrm{N}$

5- Remove more ISI in (5) by calculating

$$
\dot{\mathbf{r}}^{(K)}(i)=\left[\mathbf{r}_{r d}(1, i)-\mathbf{I}(1, i), \mathbf{r}_{r d}^{*}(2, i)-I^{*(K-1)}(2, i)\right]^{T}
$$

where

$$
\begin{aligned}
\mathbf{I}^{*(K-1)}(2, i) & =h_{2}^{*}(-1)\left[\mathbf{s}^{(K-1)}(1, i)\right]^{*} \\
& +\left(U_{2}^{*} h_{3}^{*}(-1)+h_{4}^{*}(-1)\right)\left[\mathbf{s}^{(K-1)}(2, i)\right]^{*}(11)
\end{aligned}
$$

6- Substitute $\mathbf{r}(i)$ in (8) with $\dot{\mathbf{r}}^{(K)}(i)$ to obtain $\mathbf{y}^{(K)}(i)$, and then apply the LS detection to $\mathbf{y}^{(K)}(i)$ to obtain the detection result of $\hat{\mathbf{s}}^{(K)}(j, i)$.

7- Repeat the process from point 4 until $K \leq N$

\section{SIMULATION RESULTS}

Simulation results of our proposed closed-loop EO-STBC under imperfect synchronization is given in this section. All simulations which have performed in this paper are uncoded. The bit error rate (BER) performance against signal to noise ratio (SNR) was simulated by using 8PSK gray mapping scheme in [10]. The SNR is defined as $S N R=\sigma_{s}^{2} / \sigma_{n}^{2}$ and all relay nodes transmit at $1 / 4$ power. A comparison was made between the BER performance of the proposed closed-loop EO-STBC with previous work in [10] under perfect synchronization as shown in Fig 4. In Fig. 4 and 5, the impact level of synchronization is shown by changing the value of $\beta_{m}$, that means the time delay between $R_{1}$ and the other relays is changed. Fig. 4, shows the result of conventional detector under different $\beta_{m}$ value also the BER for (DT), closed-loop EO-STBC and previous work in [10] under perfect synchronization are included as reference, Fig. 4, shows the conventional detector is not effective to synchronization error even under small time misalignments $\beta_{m}=-6$. On the other hand, the PIC scheme is very effective in mitigating synchronization error even under large time misalignments $\beta_{m}=0$ as shown in Fig (5) when the number of PIC iterations $\mathrm{K}=3$. Fig. 6, illustrates the BER of PIC iterations for $\mathrm{K}=0,1,2,3$ and $\beta_{m}=-3$, the figure shows 
the conventional detector dose not deliver the performances gain under imperfect synchronization, while the PIC scheme is very effective to mitigate the impact of imperfect synchronization, the second and third iteration deliver the performance gain.

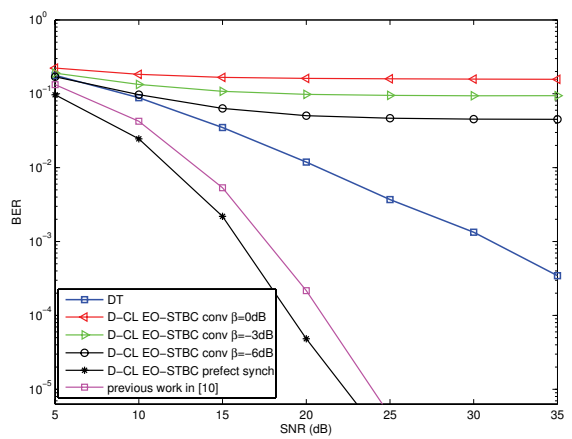

Fig. 4. The BER performance of conventional detection underdifferent $\beta_{m}$ values.

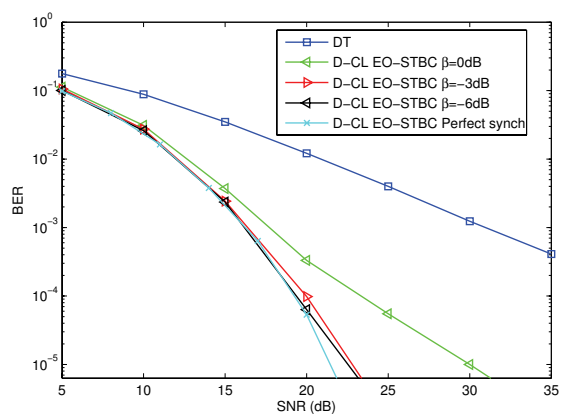

Fig. 5. The BER performance of PIC detection under different $\beta_{m}$ values.

\section{CONCLUSION}

This paper presented a simple closed-loop EO-STBC for system with four relay nodes under imperfect synchronization. To suppress the interference to the system performance, we employ the PIC detection scheme, which can effectively remove the interference caused by imperfect synchronization. Simulation results demonstrated that the proposed closed-loop EO-STBC can outperform previous work and achieve higher diversity order. Furthermore, the conventional closed-loop EO-STBC detector is very sensitive in mitigating synchronization error rather than PIC detector, Meanwhile, the proposed scheme is relatively low structural and computational complexity from previous work. Finally the received signals during phase two can be combining with received signals during phase one (DT) to deliver significant performance gain.

\section{REFERENCES}

[1] A.M. Alamouti, "A simple transmit diversity techniques for wireless communications," IEEE J, SAC, vol.16, no.8, pp.1451-1458, October 1998.

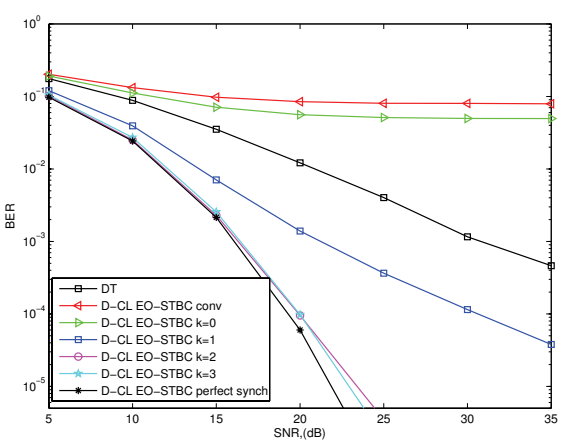

Fig. 6. The BER performance of PIC detection for different $K$ number of iterations.

[2] V. Tarokh, H. Jafarkhani, and A. Calderbank, "Space time block codes from orthogonal design," IEEE Trans Inform Theory, vol. 45, no. 5, pp.1456-1467, July 1999.

[3] A. Nosratinia, T.E Hunter,and A. Hedayat, " Cooperative communication in wireless networks," IEEE Communications Magazine, vol. 42, no. 10, pp. 74-80, October 2004.

[4] P. Mitran, H. Ochiai, and V. Tarokh, "Space-time diversity enhancements using collaborative communications," IEEE Trans Inform Theory, vol. 51, no. 6, pp.2041-2057,June 2005.

[5] J. Laneman, and G. Wornell, "Distributed SpaceTime-Coded Protocols for Exploiting Cooperative Diversity in Wireless Networks," IEEE Trans Inform Theory, vol.49, no. 10, pp. 2415-2425, October 2003.

[6] X. Li, "Space-time coded multi-transmission among distributed transmitters without perfect synchronization," IEEE Signal Processing Letters, vol. 11, no. 12, pp. 948-951,December 2004.

[7] S. Wei, D. Goeckel, and M. Valenti, "Asynchronous Cooperative Diversity," IEEE Trans on Wireless Commu, vol. 5, no. 6, pp. 1547-1557, June 2006.

[8] F. Zheng, A. Burr, and S Olafsson, "PIC detector for distributed spacetime block coding under imperfect synchronisation," IET Electronics Letters, vol. 43. no. 10, pp. 580-581, May 2007.

[9] X. Li, F. Ng, J. Hwu, and M. Chen,"Channel equalization for STBC-encoded cooperative transmissions with asynchronous transmitters" Proc. of rhe 39th Asilomar conf. on Signsls Systems and Computer,pp.457-461, October 2005.

[10] F. Zheng, A. Burr, and S. Olafsson,"Distributed Space-Time Block Coding for 3 and 4 Relay Nodes: Imperfect Synchronisation and a Solution," The 18th Annual IEEE International Symposium PIMRC,2007.

[11] Y. Yu, S. Keroueden, and J. Yuan," Closed-Loop Extended Orthogonal SpaceTime Block Codes for Three and Four Transmit Antennas," IEEE Signal Processing Letters, vol. 13, no. 5, May 2006.

[12] J. Laneman, D. Tse, and G. Wornell, "Cooperative diversity in wireless networks: efficient protocols and outage behaviour," IEEE Trans. Inform. Theory, vol. 50, no. 12, pp. 3062-3080, December 2004.

[13] F. Zheng, A. Burr, and S. Olafsson, " Signal detection for orthogonal space time block coding over time selective fading channel: a PIC approach for the Gi system" IEEE Trans. Commu, vol. 53,no. 6, pp. 969-972, June 1998. 